\title{
Design and Analysis of Folding Mechanism for a Horizontal Stabilizer in a Helicopter
}

\author{
${ }^{1}$ H.S. Karthik, ${ }^{2}$ C. Prithvi \\ ${ }^{1}$ M.Tech Student, ${ }^{2}$ Assistant Professor, Department of Mechanical Engineering, The National \\ Institute of Engg., Mysuru, India.
}

Abstract: An Advanced Light Helicopter (ALH) is a multi-role and multi-mission helicopter for army, air force, navy, coastguard and civil operations. For the navy, the ALH has to be accommodated inside the hangar in a ship's deck. The hangar doesn't accommodate the ALH and for its accommodation inside the hangar, the tail boom and horizontal stabilizer of the ALH is folded. The horizontal stabilizer is split and two machine ribs are placed at the split area where a hinge mechanism is used with a locking pin for the movement of the horizontal stabilizer (moving and non- moving segment). Two Eye and Fork End combination is used. Fork End is placed at the non-moving segment of the horizontal stabilizer, whereas Eye End is placed at the moving segment. Eye End is rotated with respect to Fork End hinge point. A detailed designing of all mechanisms involved in this context is carried out and analysis of all individual components is done. Suitable 'Airworthy' material has to be selected. A mechanism has to be developed in such a way that the locking pin has to be in its engaged condition when the ALH is in positive flight condition.

Keywords: Airworthy material, Eye End, Fork End, Folding mechanism of horizontal stabilizer, Horizontal Stabilizer.

\section{INTRODUCTION}

The foldable portion of the wing can be folded in both horizontal and vertical direction simultaneously with a single lug-rod arrangement movement. The folding wing mechanisms have been concentrated to military and navy aircrafts in order to create room for large number of aircrafts. There are two types of wing folding: Vertical fold - In this fold the wing is folded in the upward direction from a position on the wing nearer to fuselage. It requires a pushing mechanism to fold the wing along the hinge having longitudinal axis. Multiple fold: It is the type of fold in which the wing is folded multiple times to save space in vertical as well as horizontal direction [1]. Folding is closely related to the type of folding mechanism, which is determined by the number and directions of the pivot axes. This simply implies that if the folding mechanism were simple, the folding action would also be simple, whereas if the folding system were complex, the folding action would also be complicated and time consuming [2]. FEA is the most common tool for stress and structural analysis. Various fields of study are often related. Further he guided the steps involved in the finite element analysis. The basic concept behind the FEM is to replace any complex shape with the union (or summation) of a large number of very simple shapes (like triangles) that are combined to correctly model the original part. The smaller simpler shapes are called finite elements because each one occupies a small but finite sub-domain of the original part [9].

\section{LiteratURE SURVEY}

The mechanism that keeps wings in folded position and locks at unfolded position after launch must be designed. The mechanism must also withstand aerodynamic loads acting on the control surface and impact loads upon unfolding. Small space available and weight constraints make the design of such mechanism more challenging. The mechanism consists of a spring that stores necessary energy to unfold the wing and locking pin that holds wing unfolded position. Increasing stress on the structure. Design of the mechanism is optimized by iterations in such a way that it meets system requirements and design constraints [3].

The latching mechanism is the apparatus that will serve to secure the pod to the arms, while allowing the capability to release the pod from the arms. All of the components just reside in the space between each set of arm and between the mounting flanges of the pod. The mechanism must sit fully in the space between the arms and the pod flanges so that a sealed cover may be added in the future to seal the mechanism, the pod and its payload from the elements. Also, this area is used for routing the sensor power and data cables. Therefore the mechanism must not fill the entire available space between the pod surfaces and arms [4].

A locking device is a device that switches between allowing and preventing relative motion between two parts. The requirement of a locking device differs across 
applications. Mechanical locking: The position of a mechanical component determines the locking or unlocking. Mechanical locking: The position of a mechanical component determines the locking or unlocking. Examples of such components are wedges and pawls. Friction based locking: Engaging or disengaging two friction surfaces determines if the joint is locked or unlocked. Singularity locking: Singularities in mechanisms cause a transfer ratio to go to infinity [7].

FEA is the most common tool for stress and structural analysis. Various fields of study are often related. Further he guided the steps involved in the finite element analysis. The basic concept behind the FEM is to replace any complex shape with the union (or summation) of a large number of very simple shapes (like triangles) that are combined to correctly model the original part. The smaller simpler shapes are called finite elements because each one occupies a small but finite sub-domain of the original part [9].

\section{Problem Statement}

A mechanism has to be developed in such a way that the locking pin has to be in its engaged condition, when the ALH is in positive flight condition.

\section{Design Procedure}

i. Operational Requirements

- Tail boom and horizontal stabilizer is folded to accommodate on the ship deck for the navy requirements.

- Identify the area on the horizontal stabilizer to be splitted.

- At the splitted area of the horizontal stabilizer, two machine ribs are placed one at the fixed and moving part of horizontal stabilizer.

- For folding of horizontal stabilizer, two Eye and Fork End combination is used.

- Fork End is placed at fixed part of the horizontal stabilizer and Eye End is placed at moving part of the horizontal stabilizer. Eye End is rotated with respect to Fork End hinge point.

- $\quad$ Eye and Fork End is locked and unlocked with two locking pins in folding and unfolding condition.

ii. Detail designing of the mechanisms involved

- Selection of possible mechanism or group of mechanisms which will give the desired motion.

- It involves design of structural elements, reinforcements, shaping, sizing, tolerances.

iii. Analysis of forces

- Analysis of all the individual components for the Aerodynamic and Inertia loads acting on the horizontal stabilizer.

iv. Selection of suitable materials

- Airworthy materials are selected based on the induced stress value considering the suitable factor of safety.

\section{Methodology}

Detail designing of structural components, reinforcements, shaping, fix, tolerances, sizing are built separately using Siemens NX software and the parasolid solid file is imported into ANSYS 18.1 work bench software for static analysis.

A. Geometric modelling of the Horizontal stabilizer folding mechanism

- The present study is focused on the static analysis of the Horizontal stabilizer folding mechanism. The geometric model of the horizontal stabilizer folding mechanism is modeled in Siemens NX Modeling package shown in Fig. 1, Fig. 2, Fig. 3a, 3b, 4a and 4b.

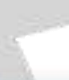



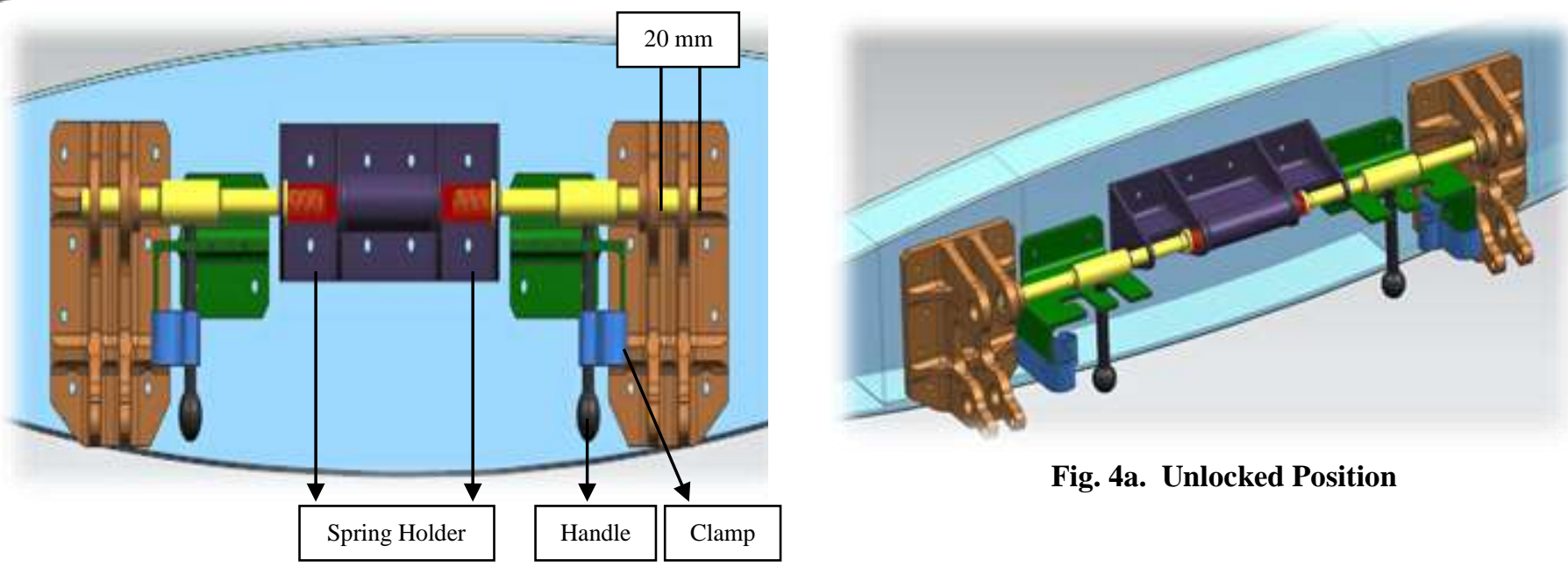

Fig. 4a. Unlocked Position

Fig. 2. Horizontal Stabilizer Folding Mechanism

- For a horizontal stabilizer to be locked the pin has to move by $20 \mathrm{~mm}$.

- The above mechanism consists of a spring holder, handle is provided with thread which is attached with the locking pin for the movement, clamp to hold the handle firmly.

- A precompression force of $10 \mathrm{~N}$ is applied to the spring to keep the locking pin always engaged with Fork and Eye end in locked position.

- The installation length of the spring is $45 \mathrm{~mm}$.

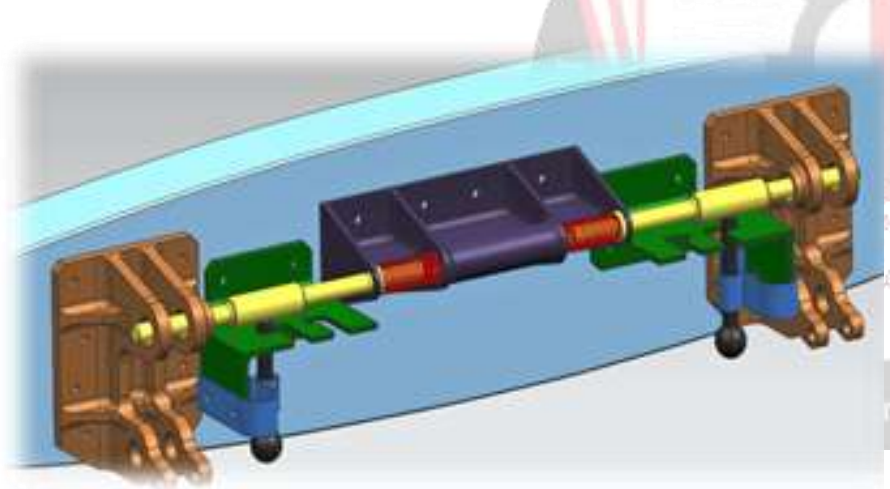

Fig. 3a. Locked Position

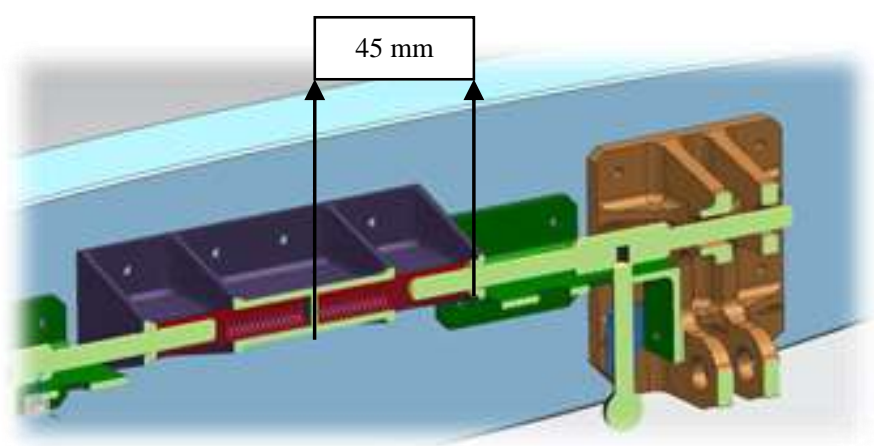

Fig. 3b. Locked Position (Sectional view)

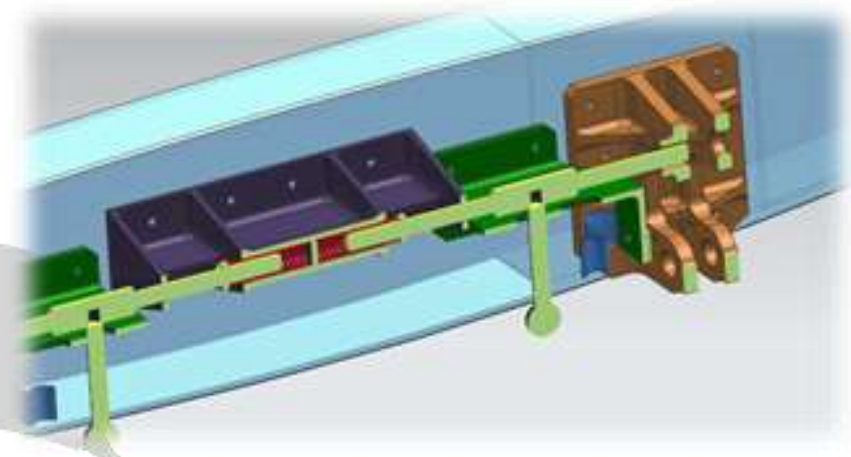

Fig. 4b. Unlocked Position (Sectional view)

- The Locked and Unlocked position of the Horizontal Stabilizer is shown in Fig. 3a and 4a.

B. Operation of the mechanism

- Pull the handle from the clamp and rotate it by $90^{\circ}$

- For a $20 \mathrm{~mm}$ axial movement move back and push down the handle to the other slot.

- The operator has to apply $15 \mathrm{~N}$ of force for $20 \mathrm{~mm}$ linear movement of the locking pin.

\section{Finite Element Modelling}

Finite element has been done for the horizontal stabilizer folding mechanism model. Initially, geometric model of horizontal stabilizer folding mechanism is modelled in Siemens NX Package is exported to ANSYS Workbench in Parasolid format Fig. 5 and 6 represents the finite element model generated after tetrahedral meshing in ANSYS. The number of Nodes is 890322 and number of elements is 547822 . The distance between the elements is considered as minimum as possible so that the results will be precise and possible.

The fixed boundary condition is considered to be at back of the machine rib. The loading is assumed to be at the tip of the locking pin. Static load is acting on the tip of the pin which is due to other parts of the horizontal stabilizer. 


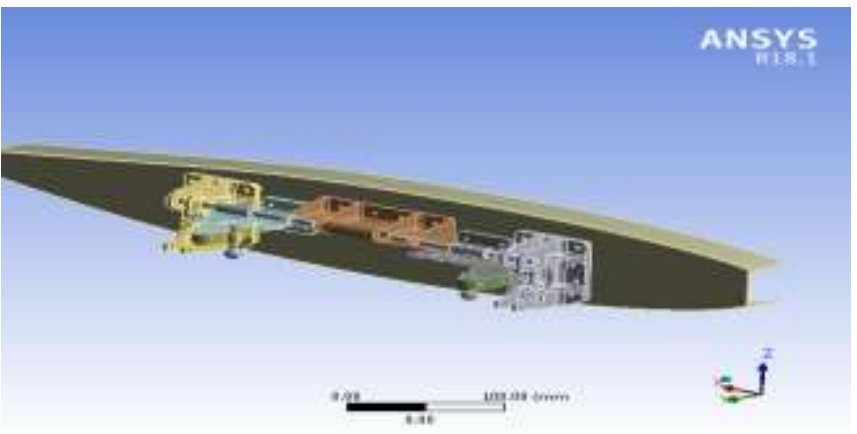

Fig. 5. Horizontal Stabilizer folding mechanism FE model

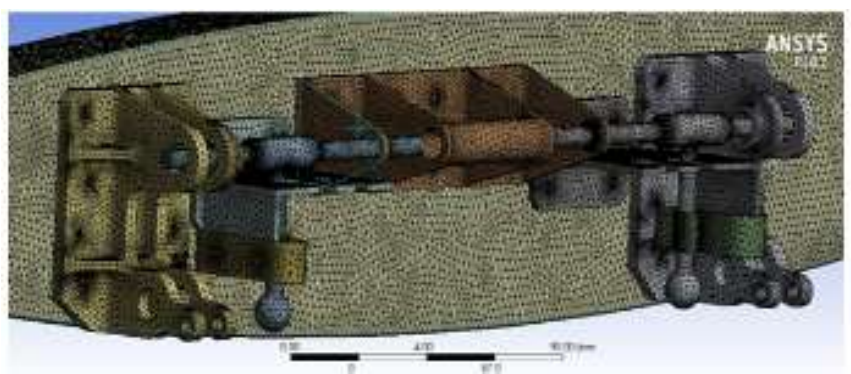

Fig. 6. Meshed model

The material properties of the components of horizontal stabilizer folding mechanism are shown in Table 1, Table 2 and Table 3 .

Table 1 Material properties of Al - Alloy

\begin{tabular}{|c|c|}
\hline Parameter & Values \\
\hline Density & $2.7 \times 10^{-6} \mathrm{~kg} / \mathrm{mm}^{3}$ \\
\hline Young's Modulus & $73 \mathrm{GPa}$ \\
\hline Poisson's Ratio & 0.32 \\
\hline
\end{tabular}

Table 2 Material properties of Stainless Steel

\begin{tabular}{|c|c|}
\hline Parameter & Values \\
\hline Density & $7.7 \times 10^{-6} \mathrm{~kg} / \mathrm{mm}^{3}$ \\
\hline Young's Modulus & $210 \mathrm{GPa}$ \\
\hline Poisson's Ratio & 0.27 \\
\hline
\end{tabular}

Table 3 Material properties of Spring Steel

\begin{tabular}{|c|c|}
\hline Parameter & Values \\
\hline Density & $7.85 \times 10^{-6} \mathrm{~kg} / \mathrm{mm}^{3}$ \\
\hline Young's Modulus & $190 \mathrm{GPa}$ \\
\hline Poisson's Ratio & 0.27 \\
\hline
\end{tabular}

\section{RESUlTS AND DISCUSSION}

A structure's static analysis is conducted to ensure that the structure is within the safety limits and to analyze the highly stressed areas in the system to minimize the chances of failure during operation. The locking pin material is chosen stainless steel hence the allowable stress for the material is $248 \mathrm{~N} / \mathrm{mm}^{2}$. From the FE model analysis, stress induced, deformation and von mises stress distribution, equivalent strain is as shown in the figure 7,8 \& 9. Maximum von mises stress induced in the structure is $157.81 \mathrm{~N} / \mathrm{mm}^{2}$.

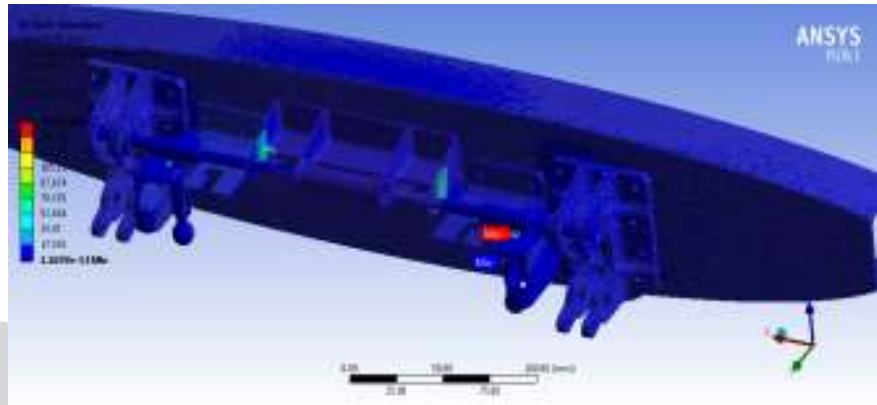

Fig. 7. Equivalent Stress (Von-Mises stress)

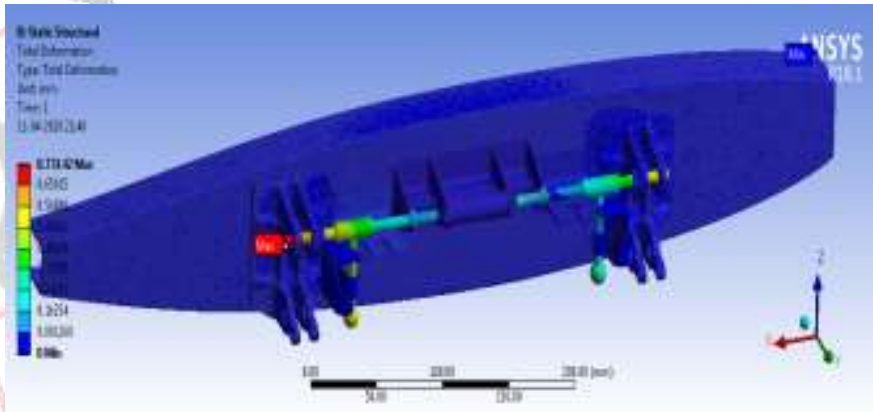

Fig. 8. Total Deformation

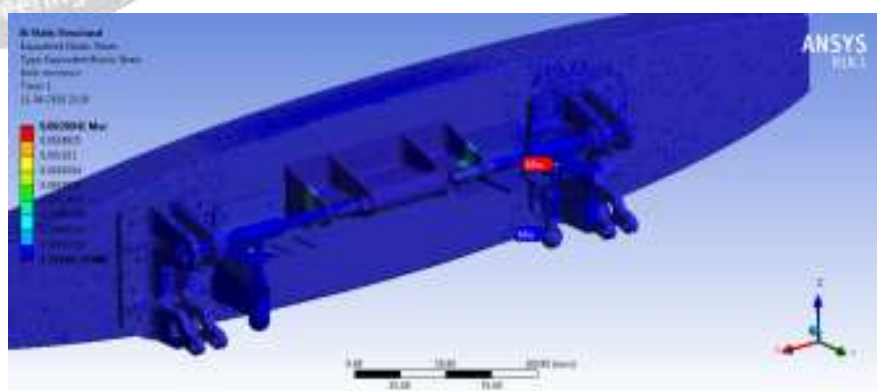

Fig. 9. Equivalent Strain

\section{CONCLUSION}

In this paper, geometric modelling and static stress analysis of horizontal stabilizer folding mechanism are presented. From the result of static stress analysis, we conclude that within the tip of the pin greatest deformation is found. On the plate highest stress and corresponding strain are observed. From the results, we infer that the structure is in safer limits as the ratio between the 
material's permissible stress and the average stress generated at any point in the entire structure is higher than 1. The mechanism is designed considering the ease of operation for the operator. The entire mechanism is inside the access cover and there is no projection of the locking pin handle. Due to this there is no accidental pushing of the locking pin handle and the operator has to open the access cover for folding and unfolding of the horizontal stabilizer. The mechanism developed is operated individually. In future a mechanism can be developed in such a way that the locking pin can be operated simultaneously.

\section{REFERENCES}

[1] Dishant Kothia, Japneet Singh \& Akanksha Dedhich "Design, Analysis and Optimization of folding wing mechanism for effective utilization of air side area" September 2016, PP 1 - 23

[2] Influence of folding mechanism of bicycles on their usability JongryunRoh, Joonho Hyeong, Sayup Kim, 2018 Human and Culture Convergence Technology Group, Korea Institute of Industrial Technology, 143 Hanggaulro, Ansan 15588, Republic of Korea, PP 1

[3] Bulent Acar, Ali Yetgin "Design and Analysis of a foldable Wing Mechanism”, PP 1- 2- 9

[4] Seth D.Lucey "Design of a quick- release mechanism for a C-130 aircraft sensor platform" 2006, PP 18

[5] Amit Geva, Hami Abramovich and Rimon Arieli "Investigation of a Morphing Wing Capable of Airfoil and span Adjustment using a Retractable Folding Mechanism"31 July 2019, PP 1-2

[6] B.S. Kiran Kumar "Pin shear- Material Testing and Validation using Finite Element Analysis (ANSYS)" July-2015, PP 1

[7] Michiel Plooij, Glenn Mathijssen and Dirk Lefeber "Review of locking devices used in robotics" March 2015, PP 1-2

[8] L.Roy, A. Sen, R.P. Chetia and M.J. Borah "Analysis and Synthesis of Four bar Mechanism” June 2008, PP 172- 173

[9] J.E. Akin "Finite Element Analysis Method" FEA Concepts: SW Simulation Overview 2009, PP 7-20

[10] E. Martinez, C. Romero, M.V. Carbonell, "On the Geometry and Design of four Bar Linkage Mechanisms" August 2014, PP 2-3

[11] Samin Akbari, Fatemeh Fallahi, Tohid Pirbodaghi "Dynamic Analysis and Controller Design for Slider crank Mechanism with Piezoelectric Actuators" May 2016

[12] Eugeny Sosnovsky, Bradleigh Windsor "CS Slide Lock Design Process Development” 2007. 